\title{
Uso de gamificação em aulas de Bioquímica como ferramenta de engajamento e motivação no ensino superior
}

\author{
Use of gamification in Biochemistry lessons as a tool for engagement and motivation in higher education
}

Yuri Rafael de Oliveira Silva ${ }^{1^{*}}$, Armando Maciel Toda $^{2}$, Seiji Isotani ${ }^{2}$, Luciana Pereira Xavier $^{1}$

${ }^{1}$ Faculdade de Biotecnologia, Instituto de Ciências Biológicas - Universidade Federal do Pará

2 Instituto de Ciências Matemáticas e de Computação - Universidade de São Paulo

(USP) - Campus de São Carlos

*e-mail: ynxs22@gmail.com

Support: CNPq, FAPESPA e FAPESP

\begin{abstract}
The traditional teaching methodology has been ineffective in stimulating the interest of students, a problem that gets worse when teaching biochemistry, which seems like an abstract and complex course. One strategy of didactic intervention that is being suggested in the past few years is Gamification. In this work, game concepts were used to develop a mechanic in which the students acquire points when they take specific actions during the semester, that can be spent in a shop that sells benefits which can affect their performances. The gamified course was applied to students of the Biotechnology major at Universidade Federal do Pará. The students' motivation was analyzed using a questionnaire, which evaluated the subjective experience of participants, that considered the course interesting, pleasant and fun. This methodology is presented as a complement to other teaching methods, with simple, flexible and costless implementation.
\end{abstract}

Keywords: Gamification; Motivation; Engagement

\section{Resumo}

A metodologia tradicional de ensino tem sido ineficaz em conquistar o interesse dos alunos, problema que se agrava no ensino da bioquímica, que aparenta ser uma disciplina abstrata e complexa. Uma das estratégias de intervenção didática que vem sendo sugerida nos últimos anos consiste na Gamificação. Neste trabalho, foram utilizados conceitos de jogos para a criação de uma mecânica onde os alunos recebem pontos por desenvolver ações durante o semestre, que podem ser gastos em uma loja que vende benefícios que podem afetar sua performance. A disciplina gamificada foi aplicada a alunos do curso de Biotecnologia da Universidade Federal do Pará. A motivação foi analisada através de questionário que avaliou a experiência subjetiva dos participantes, no qual os alunos consideraram o curso interessante, agradável e divertido. Esta metodologia apresenta-se como um complemento a outros métodos de ensino, sendo de implementação simples, flexível e sem custo.

Palavras-chave: Gamificação; Motivação; Engajamento 
Uso de gamificação em aulas de Bioquímica como ferramenta de engajamento e motivação no ensino superior

\section{Ficha da atividade}

\begin{tabular}{|c|c|}
\hline Título & $\begin{array}{l}\text { Uso de gamificação em aulas de Bioquímica como ferramenta de engajamento } \\
\text { e motivação no ensino superior }\end{array}$ \\
\hline Categoria: & $\begin{array}{l}\text { Prêmio Nacional de Ensino de Bioquímica e Biologia Molecular "Bayardo } \\
\text { Baptista Torres": Prática de Ensino }\end{array}$ \\
\hline $\begin{array}{l}\text { Tipo de } \\
\text { atividade }\end{array}$ & Intervenção metodológica/Gamificação \\
\hline Público-alvo: & Alunos da disciplina Bioquímica do Ensino Superior \\
\hline $\begin{array}{l}\text { Conteúdos } \\
\text { abordados }\end{array}$ & $\begin{array}{l}\text { Introdução à Bioquímica e Macromoléculas; Metabolismo Energético; } \\
\text { Biossíntese de Moléculas da Vida }\end{array}$ \\
\hline $\begin{array}{l}\text { Objetivos } \\
\text { educacionais }\end{array}$ & $\begin{array}{l}\text { Aumentar o engajamento e motivação de alunos na disciplina Bioquímica no } \\
\text { Ensino Superior }\end{array}$ \\
\hline $\begin{array}{l}\text { Duração } \\
\text { estimada }\end{array}$ & 6 meses (ou a duração da disciplina) \\
\hline $\begin{array}{l}\text { Materiais } \\
\text { utilizados }\end{array}$ & Usar o que informou na ficha do prêmio \\
\hline Justificativa & $\begin{array}{l}\text { O desinteresse e a falta de motivação por parte dos alunos da disciplina } \\
\text { Bioquímica são um problema recorrente, devido a conteúdos que à primeira } \\
\text { vista são abstratos e complexos. A estratégia de gamificação aplicada neste } \\
\text { estudo, visa utilizar elementos de jogos de forma a aumentar a motivação dos } \\
\text { discentes em relação ao aprendizado da Bioquímica, estimulando a iniciativa } \\
\text { autônoma dentro e fora da sala de aula. }\end{array}$ \\
\hline
\end{tabular}


Uso de gamificação em aulas de Bioquímica como ferramenta de engajamento e motivação no ensino superior

\section{Introdução}

Aulas expositivas com pouca ou nenhuma participação por parte dos aprendizes, são comuns e amplamente utilizadas nos cursos de ensino superior no Brasil, no entanto, aulas sem o planejamento de instrumentos pedagógicos falham em motivar e engajar os alunos [1]. As disciplinas de bioquímica do ensino superior costumam ser lecionadas nos primeiros semestres dos cursos, sendo apontadas pelos estudantes como complexas e difíceis [2], o que torna ainda mais difícil alcançar a motivação e engajamento dos discentes.

Dentre os instrumentos que surgiram na última década, que influenciam diretamente na motivação do aprendiz, a gamificação surgiu como o uso de elementos de jogos fora do contexto original [3], sendo amplamente utilizada em áreas como marketing, administração e saúde. Seu uso no ensino tem sido sugerido como um método de intervenção que pode ser modelado, utilizando diferentes elementos de jogos de acordo com os contextos educacionais e perfis de estudantes, tendo efeito direto na aprendizagem e desempenho dos alunos [4, 5]. Entretanto, seu uso no ensino superior tem sido limitado as ciências exatas, com escassos exemplos nas ciências biológicas [4].

A maioria das publicações científicas que enfocam o uso da gamificação no ensino descrevem mecanismos e dinâmicas de jogos e reiteram o seu uso em contexto educacional, portanto os trabalhos que efetivamente demonstram a incorporação de elementos de jogos em ambiente de ensino eram escassos [4]. Da mesma forma o uso de jogos gamificados ou simulações computacionais no ensino de áreas biotecnológicas são reportados como escassos por Bonde et al [6], estes autores no mesmo trabalho descrevem o uso de elementos de gamificação e simulação computacional de um laboratório de engenharia genética no ensino de biotecnologia, demonstrando a eficácia da estratégia pelo aumento da motivação e da melhora na aprendizagem dos alunos participantes da pesquisa.

Com o objetivo de aumentar a motivação dos discentes em relação ao aprendizado, estimulando a iniciativa autônoma dentro e fora da sala de aula, foi realizado um processo de gamificação na disciplina "Bioquímica" lecionada aos alunos do primeiro semestre do curso de Biotecnologia da Universidade Federal do Pará, aplicando o método apresentado por Toda et al [3]. Utilizando elementos de jogos como Pressão temporal, Oportunidades, Atividades Cognitivas, Competição, Níveis, Progresso, Cooperação, Aleatoriedade, Pontos e Economia, foi criado um sistema de pontuação que bonificava os estudantes que cumprissem ações pré-determinadas durante o decorrer da disciplina, 
Uso de gamificação em aulas de Bioquímica como ferramenta de engajamento e motivação no ensino superior

com pontos que poderiam ser gastos com recompensas especiais durante o semestre, por meio de um mercado interno. As ações foram definidas objetivando incentivar a participação dos alunos durante as aulas, e a dedicação extraclasse para a realização de listas de exercícios.

\section{Apresentação do material ou prática de ensino}

A conquista de pontos é guiada por regras, que definem quais ações garantem pontos e impõem limitações para que não haja exagero por parte dos participantes. Cada ação apresenta elementos de propriedade e elementos de feedback pré-definidos, que determinam seu funcionamento como mecânica de jogo (Apêndice A).

As regras utilizadas nesta aplicação são apresentadas na Figura 1, mas podem ser modificadas de acordo com a necessidade da disciplina e do regimento da instituição na qual a metodologia é aplicada. Destaca-se a importância da apresentação visual na qual a lista de regras é apresentada, pois a estética é um dos fatores responsáveis por manter os participantes interessados na experiência lúdica [7].

\begin{tabular}{|c|c|c|}
\hline Atividade & Pontuaça & Regra Assuciada \\
\hline $\begin{array}{l}\text { Entrega de lista de exercício dentro do } \\
\text { prazo estipulado }\end{array}$ & 1 ponto & $\begin{array}{l}\text { Uma lista só pode ser entregue } \\
\text { mediante a apresentação da lista } \\
\text { anterior. }\end{array}$ \\
\hline $\begin{array}{l}\text { Participar da atividade de jogos } \\
\text { educacionais em grupo na data } \\
\text { marcada }\end{array}$ & $\begin{array}{l}3 \text { pontos (Primeiro lugar) } \\
2 \text { pontos (Segundo lugar) } \\
1 \text { ponto (Terceiro lugar) }\end{array}$ & $\begin{array}{l}\text { A pontuação será recebida por } \\
\text { cada membro do grupo. }\end{array}$ \\
\hline Ter $100 \%$ de frequência na disciplina & 1 ponto & $\begin{array}{l}\text { É necessário ter no mínimo } 75 \% \\
\text { de frequência para ser aprovado } \\
\text { na disciplina }\end{array}$ \\
\hline $\begin{array}{l}\text { Participar em sala de aula na forma } \\
\text { de perguntas, observações e } \\
\text { discussões }\end{array}$ & 1 ponto & $\begin{array}{l}\text { O docente é responsável por } \\
\text { decidir se a participação foi } \\
\text { relevante }\end{array}$ \\
\hline
\end{tabular}

As pontuações não são transferíveis entre os alunos participantes.

Figura 1. Lista de regras utilizadas na aplicação do método gamificado na turma de Bioquímica do curso de Biotecnologia da Universidade Federal do Pará.

O docente é responsável por garantir o cumprimento das regras e manter um Mapa de Progresso, onde é possível conferir a pontuação atual de cada aluno. Os pontos podem ser gastos em uma Loja, que concede regalias na disciplina para o indivíduo ou para o grupo de participantes, como apresentado na Figura 2. 
Uso de gamificação em aulas de Bioquímica como ferramenta de engajamento e motivação no ensino superior
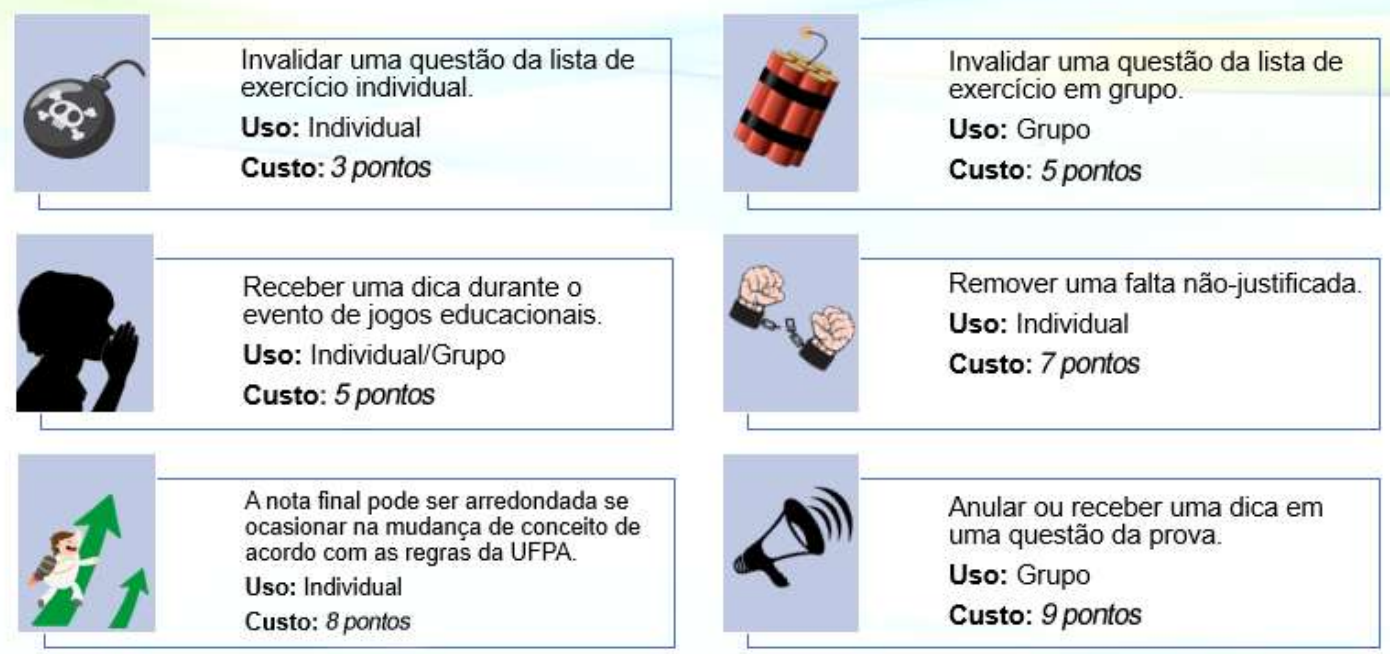

Figura 2. Loja de regalias, onde a moeda de troca são pontos conquistados no decorrer da disciplina.

\section{Potencial Educacional}

O uso da gamificação com o propósito de resolver problemas e instigar questionamentos apresenta-se como uma excelente metodologia facilitadora do ensinoaprendizagem: a) No aspecto cognitivo, promove a motivação e o engajamento dos envolvidos, auxiliando-os na exploração e descoberta, incentivando o planejamento e execução de tarefas para alcançar os objetivos propostos; b) No aspecto emocional, instiga a curiosidade, a competição e cooperação, a descoberta do potencial individual pelo próprio sujeito e favorece a oportunidade de superar e entender as falhas individuais como parte essencial do ciclo de aprendizagem; c) No aspecto social, reforça o papel do indivíduo em um grupo, reconhecimento e inserção social no ambiente de ensino [8].

$\mathrm{Na}$ experiência de ensino gamificado de Bioquímica, foi possível observar o aumento da participação voluntária dos alunos e a motivação e criatividade nos trabalhos propostos. Também foi notada uma maior interação entre os participantes promovendo socialização, a apresentação de redações e respostas mais elaboradas e/ou aprofundadas, além do sucesso acadêmico.

$\mathrm{Na}$ avaliação da disciplina pelo ponto de vista dos alunos, realizada através de um questionário que considerou o interesse e aproveitamento, a competência percebida, a pressão e tensão, e a escolha percebida, a experiência foi considerada agradável, interessante e divertida (Apêndice B).

Portanto, o uso da gamificação no ensino de Bioquímica se apresenta com grande potencial educacional, por facilitar e incentivar o desenvolvimento dos alunos nos aspectos cognitivo, social e emocional em ambiente descontraído e motivador. 


\section{Diferencial com os materiais/ atividade pré-existentes}

Este trabalho apresenta a primeira aplicação publicada do uso da gamificação na disciplina Bioquímica no ensino superior, e uma das primeiras na área de ciências biológicas. Outro diferencial é que este método não requer o uso de recursos computacionais, como ocorre com grande parte dos processos gamificados na literatura presentes na literatura.

As atividades pré-existentes utilizadas e publicadas nos últimos anos, visando o aumento na motivação e engajamento dos alunos na bioquímica, envolvem majoritariamente o uso de jogos sérios e a aplicação de aulas práticas. Ambos costumam ser focados em tópicos específicos da disciplina, sendo aplicados apenas uma vez durante o decorrer do período letivo, motivando os discentes por um período curto de tempo. O método descrito neste artigo, engloba todo o período e tópicos da disciplina de forma continuada.

\section{Procedimentos de uso ou aplicação}

O primeiro passo a ser executado pelo docente é a elaboração das regras de pontuação, para isso, é necessário realizar um mapeamento das ações executadas pelos estudantes. Estas ações são parte do comportamento esperado pelo docente, e envolvem interações como: fazer uma pergunta pertinente no meio da explicação. Cada ação mapeada é então associada a uma pontuação, gerando uma regra. As regras devem ter limitações de maneira a não tornar a experiência desmotivadora para os participantes. Em seguida são definidas as recompensas que poderão ser adquiridas pelos alunos na loja. Os itens da loja, principais motivadores para a obtenção de pontos, devem conceder benefícios que não poderiam ser obtidos na disciplina utilizando o método regular de ensino, e seus valores devem ser balanceados de acordo com a quantidade total de pontos que podem ser obtidos. O máximo de pontos que podem ser obtidos pode ser calculado a partir do cronograma da disciplina e das regras de pontuação.

No início do período letivo, a atividade deve ser explicada para os discentes, fornecendo a opção de participar da mesma ou realizar apenas a avaliação tradicional. No decorrer da disciplina, o docente é responsável por registrar as pontuações, garantir o cumprimento das regras, e permitir que os alunos sejam capazes de acompanhá-las através de um mapa de progresso. A loja pode ser disponibilizada continuamente ou em períodos específicos, a depender de quais itens são oferecidos. 
Uso de gamificação em aulas de Bioquímica como ferramenta de engajamento e motivação no ensino superior

\section{Considerações finais}

Este trabalho apresentou um modelo do uso de gamificação em aulas de bioquímica, sendo um dos primeiros na área de ciências biológicas. A disciplina aplicada com o uso da gamificação foi eficaz em tornar as aulas mais participativas, incentivando o interesse e a motivação dos alunos nos questionamentos em sala de aula e na entrega das listas de exercícios.

A disciplina gamificada apresentada pode ser facilmente adaptada de acordo com as necessidades de cada turma e professor, compensando por algumas desvantagens do método de ensino tradicional sem mudanças radicais e sem custo financeiro.

\section{Referências}

[1] Gil ES, Garcia EYA, Lino FMA, Gil JLV. Estratégias de Ensino e Motivação de Estudantes no Ensino Superior. Vita et Sanitas. 2012;6:57-81.

[2] Silveira JT, Rocha JBT. Produção científica sobre estratégias didáticas utilizadas no ensino de Bioquímica: uma revisão sistemática. Revista de Ensino de Bioquímica. 2016;14(1):7-21.

[3] Toda AM, Silva YRO, Cruz W, Xavier L, Isotani S. Um processo de Gamificação para o ensino superior: Experiências em um módulo de Bioquímica. Anais do Workshop de Informática na Escola 2016.

[4] Dicheva D, Dichev C, Agre G, Angelova G. Gamification in Education: A Systematic Mapping Study. Journal of Educational Technology \& Society. 2015;18(3):75-88.

[5] Fardo ML. A gamificação aplicada em ambientes de aprendizagem. Revista Novas Tecnologias na Educação. 2013;14(1).

[6] Bonde M, Makransky G, Wandall J, Larsen M, Morsing M, Jarmer H et al. Improving biotech education through gamified laboratory simulations. Nature Biotechnology. 2014;32(7):694-697.

[7] Kapp KM. The gamification of learning and instruction. 1st ed. San Francisco: Pfeiffer, p.46; 2012.

[8] Lee JJ, Hammer J. Gamification in Education: What, How, Why Bother? Academic Exchange Quarterly. $2011 ; 15(2)$. 
Uso de gamificação em aulas de Bioquímica como ferramenta de engajamento e motivação no ensino superior

Apêndice A. Lista de ações

Ação 1: Entrega da lista de exercícios individual

Descrição: Será aplicada uma lista de exercícios, sendo resolvida individualmente. Ao final, será atribuído um número de pontos baseado na correção da lista.

\section{Elementos de propriedade:}

PRESSÃO: Data de entrega sem prorrogação

OPORTUNIDADES: Todos os alunos têm acesso às mesmas questões nas listas

ATIV. COGNITIVAS: Exercícios

Elementos de feedback:

NÍVEIS: Uma lista é pré-requisito da anterior

PROGRESSO: Mapa de progresso

PONTOS/RECOMPENSA: Nota e pontuação bônus (economia)

Período / módulo: Final de cada tópico

Ação 2: Entrega da lista de exercícios em grupo

Descrição: Será aplicada uma lista de exercícios, sendo resolvida em grupo. Ao final, será atribuída a nota baseando-se na correção da lista.

\section{Propriedades:}

COOPERAÇÃO: Listas em grupos de $\mathrm{N}$ alunos

ALEATORIEDADE: Questões aleatórias nas listas (Utilizando um banco de questões)

COMPETIÇÃO: Grupos competindo entre si

PRESSÃO: Data para entrega das listas

\section{Feedback:}

NÍVEIS: Uma lista é pré-requisito da anterior

PROGRESSO: Mapa de progresso

PONTOS/RECOMPENSA: Pontuação bônus (economia)

Período / módulo: Uma lista exercício antes da primeira prova, duas antes da segunda.

Ação 3: Participação no evento de jogos educacionais

Descrição: Um dia reservado para uso de jogos lúdicos a serem praticados em grupo que servirá como revisão para a primeira prova. A pontuação do grupo contará para o placar.

\section{Propriedades:}

COOPERAÇÃO: Alunos discutindo estratégias em grupo. 
COMPETIÇÃO: Grupos competindo entre si

PRESSÃO: Tempo limite dos jogos.

ATIV. COGNITIVA: Cada jogo explorando diferentes habilidades do aluno.

\section{Feedback:}

PONTOS: Cada grupo ganhará uma pontuação dependendo de sua colocação no evento.

Período / módulo: Na aula anterior à primeira prova.

Ação 4: Compras na loja

Descrição: Utilização dos pontos obtidos para adquirir as vantagens oferecidas pela loja.

Propriedades:

COOPERAÇÃO: Alguns itens da loja oferecem vantagens em grupo.

DECISÕES: As pontuações não permitem a compra de todos os itens. É necessário escolher.

OPORTUNIDADE: Todos têm acesso aos mesmos itens.

\section{Feedback:}

CONQUISTAS: Os itens servem como recompensa pelas atividades exercidas para obtenção da pontuação.

Período / módulo: No decorrer do semestre. 
Uso de gamificação em aulas de Bioquímica como ferramenta de engajamento e motivação no ensino superior

Apêndice B. Avaliação da aplicação

A atividade foi avaliada através de aplicação do Intrinsic Motivation Inventory (IMI) para medir a motivação dos discentes, e examinar se o processo obteve o efeito engajador desejado. Este questionário visa identificar certos aspectos relacionados a motivação do indivíduo, através de uma escala Lickert de 7 itens, onde 1 significa "Não é verdade" e 7 significa "Totalmente verdade".

O questionário utilizado neste estudo foi construído com 21 questões, sendo aplicado e preenchido de forma manual (papel e caneta) ao fim da disciplina, de forma anônima, abrangendo perguntas dos grupos: (A) Interesse/Aproveitamento, (B) Competência Percebida, (C) Pressão/Tensão e (D) Escolha Percebida. Para analisar os resultados coletados, foi considerada a média $(\mu)$ das respostas, considerando o gênero identificado pelo participante no questionário. Os resultados são apresentados no Quadro 1.

Foram avaliados 24 participantes, sendo 13 do sexo masculino e 11 do sexo feminino. No geral, os resultados que mais se afastam do ponto central (Pouco verdade, 4) foram das perguntas do grupo (Interesse/Aproveitamento), nas quais os alunos consideraram o curso agradável (P20: $\mu($ Geral $)=5,0)$, interessante $(\mathrm{P} 5: \mu($ Geral $)=5,5)$ e divertido (P8: $\mu($ Geral) $=5,7)$, além de não considerarem chato $(\mathrm{P} 14: \mu(\mathrm{Geral})=2,1)$. Quanto as diferenças entre os sexos, as participantes do sexo feminino gostaram mais do curso (P10: $\mu(\mathrm{F})>\mu(\mathrm{M})$, dif:1,0), sentiram que estavam fazendo o que queriam (P15: $\mu(\mathrm{F})>\mu(\mathrm{M})$, dif:1,0) e tiveram maior interesse (P17: $\mu(\mathrm{F})>\mu(\mathrm{M})$, dif:1,0). Já os participantes do sexo masculino se sentiram mais obrigados a fazer atividade $(\mathrm{P} 19: \mu(\mathrm{M})>\mu(\mathrm{F})$, dif:1,5) e se sentiram menos competentes que as do sexo feminino (P16: $\mu(\mathrm{F})>\mu(\mathrm{M})$, dif:0,7). 
Uso de gamificação em aulas de Bioquímica como ferramenta de engajamento e motivação no ensino superior

Quadro 1. Perguntas* do IMI aplicado e medias por gênero.

\begin{tabular}{|c|c|l|c|c|c|}
\hline ID & Grupo & \multicolumn{1}{|c|}{ Pergunta } & $\mu(\mathrm{F})$ & $\mu(\mathrm{M})$ & $\mu(\mathrm{Geral})$ \\
\hline P1 & A & $\begin{array}{l}\text { Enquanto eu estava fazendo a atividade, eu } \\
\text { estava pensando o quanto estava aproveitando. }\end{array}$ & 4,1 & 3,8 & 4,0 \\
\hline P2 & C & $\begin{array}{l}\text { Eu não me senti nervoso(a) enquanto fazia a } \\
\text { atividade. }\end{array}$ & 4,9 & 4,8 & 4,8 \\
\hline P3 & C & $\begin{array}{l}\text { Eu senti que foi minha escolha fazer essa } \\
\text { atividade. }\end{array}$ & 4,7 & 4,4 & 4,5 \\
\hline P4 & B & Eu acho que sou muito bom nessa atividade. & 3,5 & 3,8 & 3,7 \\
\hline P5 & A & Eu achei essa atividade muito interessante. & 6,0 & 5,2 & 5,5 \\
\hline P6 & C & Eu me senti tenso durante essa atividade. & 2,9 & 3,3 & 3,1 \\
\hline P7 & B & $\begin{array}{l}\text { Eu acho que me saí bem nessa atividade } \\
\text { comparado a outros estudantes. }\end{array}$ & 4,3 & 3,7 & 4,0 \\
\hline P8 & A & Fazer essa atividade foi divertido. & 6,0 & 5,4 & 5,7 \\
\hline P9 & C & Eu me senti tranquilo(a) fazendo essa atividade. & 3,8 & 4,2 & 4,0 \\
\hline P10 & A & Eu gostei muito de fazer essa atividade. & 5,2 & 4,2 & 4,7 \\
\hline P11 & D & $\begin{array}{l}\text { Eu não tive muita escolha em fazer essa } \\
\text { atividade. }\end{array}$ & 3,2 & 4,0 & 3,6 \\
\hline P12 & B & $\begin{array}{l}\text { Eu estou satisfeito(a) com o meu desempenho } \\
\text { nessa atividade. }\end{array}$ & 4,4 & 4,5 & 4,4 \\
\hline P13 & C & Eu estava ansioso(a) durante a atividade. & 3,8 & 3,3 & 3,5 \\
\hline P14 & A & Eu achei essa atividade muito chata. & 1,9 & 2,2 & 2,1 \\
\hline P15 & D & $\begin{array}{l}\text { Eu senti que estava fazendo o que eu queria } \\
\text { enquanto eu estava fazendo a atividade. }\end{array}$ & 4,6 & 3,6 & 4,1 \\
\hline P16 & B & Me senti muito competente nesta atividade. & 4,4 & 3,7 & 4,0 \\
\hline P17 & A & Eu achei a atividade muito interessante. & 6,0 & 5,0 & 5,5 \\
\hline P18 & C & $\begin{array}{l}\text { Eu me senti pressionado enquanto fazia a } \\
\text { atividade. }\end{array}$ & 3,7 & 3,2 & 3,5 \\
\hline P19 & D & Eu senti obrigação de fazer essa atividade. & 3,0 & 4,5 & 3,8 \\
\hline P20 & A & $\begin{array}{l}\text { Eu descreveria essa atividade como muito } \\
\text { agradável. }\end{array}$ & 4,6 & 5,3 & 5,0 \\
\hline P21 & D & Eu fiz essa atividade porque não tive escolha. & 3,0 & 3,2 & 3,1 \\
\hline P22 & B & $\begin{array}{l}\text { Depois de fazer essa atividade por um tempo, } \\
\text { me senti muito competente. }\end{array}$ & 4,9 & 4,1 & 4,5 \\
\hline
\end{tabular}

* "Para cada frase, indique o quão verdadeiro é para você, utilizando a seguinte escala: 1 para não é verdade, 4 para um pouco verdade, 7 para verdade. Considere a aula de Bioquímica como a atividade nas perguntas. " 